Extensive recombination in a population can also limit inferences about phylogenetic history. Here, we investigate the impact of recombination in the study of isolates with reduced susceptibility to cefixime (cef ${ }^{\mathrm{RS}}$; cefixime MICs $\geq 0.25 \mu \mathrm{g} / \mathrm{ml}$ ) in the United States.

Methods We generated draught genome sequences for 242 gonococcal isolates collected by CDC's Gonococcal Isolate Surveillance Program (GISP). These isolates comprise all 141 cef $^{\text {RS }}$ isolates from GISP in 2009-10 and 141 susceptible isolates matched by location, collection date, and sexual orientation of the infected individual. We predicted recombinant regions and generated a maximum likelihood phylogenetic tree from core SNPs. We performed in silico MLST and NG-MAST typing, and compared phylogenies of antibiotic resistance loci to whole genome-based phylogenies.

Results Per site $r / m$ ratios (relative likelihood that a polymorphism was introduced through recombination rather than mutation) of recent branches in the phylogenetic tree are higher and fraction of homoplasic sites much lower than for the overall tree, suggesting that extensive recombination reduces confidence in the phylogeny's deep branches. Comparison with in silico MLST and NG-MAST reveals that traditional typing-based phylogenetic inferences, even for recent events, are confounded by recombination. Of the 21 penA alleles in this dataset, mosaic PBP2 pattern XXXIV was the most common (present in $116 / 121$ cef $^{\mathrm{RS}}$ isolates). We find several recombination events introducing this allele into distinct lineages, and an event within the $d c w$ gene cluster, which includes the penA allele, associated with reversion from cef ${ }^{\mathrm{RS}}$ to cefixime susceptibility.

Conclusions Genomic methods reveal the impact of recombination on phylogenetic history, spread of resistance elements, and genome evolution, and offer a superior approach to traditional typing schemes in understanding population structure and dynamics.

\section{YI.5 SEXUAL RISK FACTORS AMONGST WOMEN WHO HAVE SEX WITH MEN AND WOMEN: THE WOMEN IN INDIANA SEXUAL HEALTH AND EXPERIENCE STUDY (WISHES)}

doi:10.1136/sextrans-2013-051184.0082

V R Schick, B Van Der Pol, B Dodge, A Bell, C Neal. Indiana University, Bloomington, IN, United States

Background Previous research suggests that women who have sex with women and men (WSWM) may be at a higher risk for STI than women with exclusively male (WSM) or female (WSW) partners. In contrast to previous research which has compared WSWM to WSW/WSM, the present study explored risk factors which may be unique to WSWM. Methods Local women aged 18 or older who reported genital contact with a male and female partner within the past year were invited to participate in the study. Upon completion of an internetbased baseline survey about their sexual and STI history, participants were invited to participate in an in-person interview. During the meeting, participants engaged in a semi-structured interview followed by completion of a sexual event history calendar (SEHC). Participants were then asked to self-collect oral, vaginal and anal samples to screen for Chlamydia, Gonorrhea and Trichomoniasis.

Results Eighty participants with a mean age of $26.74(\mathrm{SD}=7.97)$ completed the survey. The majority of the participants reported genital contact at an earlier age with a male partner $(M=16.02$, $\mathrm{SD}=3.31)$ than with a female partner $(M=18.93, \mathrm{~N}=5.13)$. The most common type of genital contact was external genital rubbing with a male $(97.5 \%, \mathrm{~N}=78)$ and/or female $(93.5 \%, \mathrm{~N}=74)$ partner. Most participants reported a wide variety of sexual behaviours. Over half of the participants $(57.5 \%, \mathrm{~N}=44)$ reported engaging in a threesome/orgy within the past year. Data on the sequence of sexual acts was captured using the SEHC. Most participants self-collected a sample for STI screening. Approximately $30 \%(\mathrm{~N}=25)$ of the participants reported an STI diagnosis within their lifetime and close to $10 \%$ of the participants tested positive for Chlamydia.
Discussion The study was successful in recruiting a sizable number of participants with a range of sexual experiences. The majority of participants opted to participate in all phases of the study.

\section{Oral sessions}

\subsection{1 - Microbial virulence and host response}

\subsection{ASSOCIATION OF GENETIC VARIANTS WITH CHLAMYDIA TRACHOMATIS REINFECTION}

doi:10.1136/sextrans-2013-051184.0083

R Kapil, J Tang, C G Press, W M Geisler. University of Alabama at Birmingham, Birmingham, AL, United States

Background Up to $20 \%$ of Chlamydia trachomatis (CT)-infected patients are reinfected within months after treatment, suggesting some fail to develop protective immunity. Genetic determinants influencing CT reinfection risk have not been fully elucidated. Our primary research objective is to identify genetic determinants of CT reinfection. Based on previously reported associations of HLA class II alleles with CT complications, our initial investigations focus on HLA class II genes.

Methods In an ongoing prospective natural history study, CTinfected subjects are enrolled, treated with azithromycin $1 \mathrm{~g}$ single dose, and return for a 6-month follow-up visit for repeat CT testing using the Gen-Probe APTIMA Combo 2 assay (Gen-Probe, Inc., San Diego, CA). HLA class II alleles are resolved by a combination of PCR-based techniques. Genomic DNA is stored for further genotyping.

Results A total of 199 African American subjects have been studied to date: $90 \%$ women and median age 23 . CT reinfection at follow-up was noted in $18 \%$. Subjects with $H L A-D Q B 1^{*} 05$ more often had reinfection $(20[26 \%]$ vs. $16[13 \%], \mathrm{P}=0.018)$, which remained significant after controlling for age and gender (OR 2.6, 95\% CI 1.2-5.6, $\mathrm{P}=0.012$ ). Other HLA-DQB1 alleles were not significantly associated with reinfection $(\mathrm{P} \geq 0.1)$.

Conclusion $H L A-D Q B 1^{*} 05$ was associated with CT reinfection, suggesting it could influence protective immunity. More comprehensive genotyping from larger prospectively studied cohorts should help confirm or refine this observation. Analysis of additional HLA class II genes and genes beyond the human MHC is in progress.

\subsection{INNATE IMMUNITY MODULATION BY TRICHOMONAS VAGINALIS GALECTIN-BINDING GLYCOLIPID DOMAINS}

doi:10.1136/sextrans-2013-051184.0084

${ }^{1,2} \mathbf{R}$ N Fichorova, 'H S Yamamoto, 'T Fashemi, ${ }^{10} \mathrm{R}$ Buck, ${ }^{1} \mathrm{E}$ Foley, ${ }^{3} \mathrm{G}$ R Hayes, ${ }^{4} \mathrm{~S}$ Sato, ${ }^{3 B} \mathrm{~N}$ Singh. 'Laboratory of Genital Tract Biology, Department of Obstetrics, Gynecology and Reproductive Biology, Brigham and Women's Hospital, Boston, MA, United States; ${ }^{2}$ Harvard Medical School, Boston, MA, United States; ${ }^{3}$ Department of Biochemistry and Molecular Biology and Department of Obstetrics and Gynecology, SUNY Upstate Medical University, Syracuse, NY, United States; ${ }^{4}$ Research Centre for Infectious Diseases, Faculty of Medicine, Laval University, Quebec, OC, Canada

Background Trichomonas vaginalis is a protozoan extracellular parasite causing long-lasting and recurrent vaginitis with a wide range of symptoms and increased risk of HIV and other viral STIs. The protozoan virulence factors that subvert the mucosal immune response are poorly understood. Here we investigate the role of the ceramide-phosphatidyl-inositol glycolipid core (CPI-GC) of the protozoan lipophosphoglycan (LPG), which is the major glycoconjugate on the trichomonad surface (2-3 million copies/parasite). We have previously determined that CPI-GC lacks mannose but 